\title{
Índice de masa corporal en militares y otros indicadores antropométricos
}

https://doi.org/10.21830/9789585241466.01

\author{
Maritza Gómez Leguizamón ${ }^{1}$ \\ Mercedes Mora Plazas ${ }^{2}$
}

\section{Resumen}

Objetivo: establecer la pertinencia del Índice de Masa Corporal (IMC) como estid mador de adiposidad (sobrepeso y obesidad) en población militar y su relación con otros indicadores antropométricos. Materiales y métodos: se realizó un estudio transversal con 137 militares en formación, pertenecientes a diferentes escuelas del Ejército Nacional de Colombia. Se recolectó información sociodemográfica como edad, sexo, escuela del Ejército a la que pertenecían los participantes y se realizó una evaluación antropométrica que incluyó medición de talla, peso y circunferencia de cintura. También se evaluó la composición corporal por medio de bioimpedancia eléctrica (Seca BmCA 550) y se realizó una descripción de la prevalencia de sobrepeso y obesidad. Se definieron coeficientes de correlación y se estableció la concordancia diagnóstica usando el índice Kappa de Cohen, la sensibilidad y especificidad del IMC frente a indicadores considerados como gold estándar de la composición corporal como el Porcentaje de Grasa Corporal (\%GC) y el Índice de Masa Grasa (IMG). Resultados: la correlación entre el IMC y el \%GC fue positiva $(\mathrm{r}=0,750, p=0,000)$ y superior con el IMG $(0,836, p=0,000)$. Por su parte, la correlación entre el IMC y la Masa Libre de Grasa (MLG) fue positiva débil $(0,375, \mathrm{p}=0,000)$; el Índice de Cintura-Altura (ICA) mostró la mejor correlación con \%GC e IMG. La concordancia del IMC con el \%GC ( $\mathrm{r}=0,750, p=0,000)$ y el IMG $(0,836, p=0,000)$ fue positiva. Conclusiones: si bien el IMC presentó una buena concordancia con el \%GC y el IMG, sobreestimó la prevalencia de sobrepeso

1 Grupo de Investigación Renfimil, Escuela Militar de Cadetes "General José María Córdova”. Nutricionista Dietista. Msc. Nutrición Deportiva y Clínica. Antropometrista nivel 1 ISAK. Contacto: leidy.gomez@esmic.edu.co. https://orcid.org/0000-0001-6007-0420

2 Universidad Nacional de Colombia, sede Bogotá. Msc. Nutrición Humana y Msc. Fisiología. Antropometrista Nivel 3 ISAK. Contacto: mmorap@unal.edu.co. https://orcid.org/0000-00015396-8726 
y, en menor proporción, subestimó la prevalencia de obesidad con una alta sensibilidad, pero con baja especificad para el diagnóstico de sobrepeso, así como con una alta especificidad y sensibilidad para la obesidad. Aunque el IMC muestra una correlación positiva con indicadores de adiposidad, no puede considerarse el indicador adecuado para el diagnóstico de sobrepeso y obesidad en población militar, ya que presenta falsos positivos para sobrepeso y falsos negativos para obesidad. El IMG es el indicador que mejor predice la adiposidad corporal, junto con el ICA. Se debe considerar el uso del Índice de Masa Libre de Magra (FFMI, por su sigla en inglés) como parámetro para la medición de este componente corporal, así como usar en futuras investigaciones el método antropométrico con fraccionamiento por cinco componentes, el cual tiene un valor predictivo de la composición corporal superior en población físicamente activa.

Palabras clave: índice de masa corporal, porcentaje de grasa corporal, índice de masa grasa, indicadores de adiposidad, bioimpedancia eléctrica, antropometría, compoz sición corporal.

\section{Introducción}

Durante su periodo de formación, el personal militar está sometido a múltiples actividades y esfuerzos físicos relacionados con su desempeño cotidiano, así como con sus rutinas de entrenamiento y cursos de combate. Estas actividades exigen que el militar mantenga un buen estado físico y una adecuada composición corporal, aspecto que está íntimamente relacionado con un mejor rendimiento físico (1). Así mismo, de acuerdo con el primer Programa de Fomento del Ejercicio y Hábitos Alimentarios Saludables del Departamento de Defensa de los Estados Unidos, el mantenimiento de una adecuada composición corporal no solo forma parte integral de la condición física, sino que además tiene un papel importante en la apariencia militar profesional, además de que es un buen indicador de la salud general y el bienestar del personal militar (2).

Particularmente, en el contexto militar colombiano la evaluación de las condiciones físicas toma como referencia el Índice de Masa Corporal (IMC) para clasificar el sobrepeso y la obesidad (3). Sin embargo, probablemente el uso del IMC como único indicador de estas condiciones no sea adecuado porque el entrenamiento físico de los militares genera alto desarrollo de la 
masa magra (MM), de tal manera que pueden existir casos en los que la evaluación reporta un alto IMC (sobrepeso u obesidad), cuando en realidad se trata de una alta MM, lo que se considera un falso positivo. También hay casos en los que reporta normalidad según el IMC, pero un análisis más detallado evidencia que la persona tiene una alta masa grasa (MG) en su composición corporal, lo cual se considera un falso negativo. Por esta razón surge la importancia de definir variables que faciliten la identificación del IMC por alta MM o por alta MG, así como de emplear métodos validados de fácil acceso y uso como la bioimpedancia eléctrica y la antropometría.

El propósito de este estudio es establecer la pertinencia de uso del IMC como único indicador del estado nutricional en población militar en formación. La investigación busca evaluar la utilización de otros indicadores adicionales que permitan diferenciar militares con alta masa magra o alta masa grasa, correlacionando el IMC con variables como el porcentaje de grasa, el Índice de Masa Grasa, el Índice de Masa Magra, el perímetro de la cintura y el Índice de Cintura / Altura.

\section{El IMC como indicador del sobrepeso y obesidad en población militar}

El Índice de Masa Corporal fue diseñado en 1832 por Adolphe Quetelet (4) y corresponde a un indicador global del estado nutricional que establece una relación entre peso $(\mathrm{kg})$ y el cuadrado de la estatura $(\mathrm{m})(\mathrm{IMC}=$ peso $\mathrm{kg}$ $/ \mathrm{m}^{2}$ ). Se trata de una herramienta económica, simple y rápida, ampliamente usada para determinar sobrepeso y obesidad, que tiene los siguientes puntos de corte según la Organización Mundial de la Salud (OMS): normal 18,5 kg $/ \mathrm{m}^{2}$ a $24,9 \mathrm{~kg} / \mathrm{m}^{2}$; sobrepeso $25 \mathrm{~kg} / \mathrm{m}^{2}$ a $29,9 \mathrm{~kg} / \mathrm{m}^{2}$, y obesidad > $30 \mathrm{~kg}$ $/ \mathrm{m}^{2}(4)$. Según este mismo organismo, el sobrepeso y la obesidad se definen como una acumulación anormal o excesiva de grasa que puede ser perjudicial para la salud. En el año 2016, el 39 \% de las personas mayores de 18 años en el mundo presentaron sobrepeso y el $13 \%$ obesidad (4). En Colombia, de acuerdo con cifras reportadas por la Encuesta Nacional de Alimentación 
y Nutrición (Ensin) 2015, el 56,4 \% de la población adulta (18 a 64 años) presenta exceso de peso (37,7 \% sobrepeso y $18,7 \%$ obesidad), siendo mayor en mujeres (59,6 \%) que en hombres (52,7 \%) (5). Este panorama parece no ser ajeno al personal militar en el mundo y en Latinoamérica (6-8).

En el contexto colombiano, por ejemplo, en un estudio realizado se identificó que de acuerdo con el IMC el 68,9 \% y 8,7 \% presentaban sobrepeso y obesidad, respectivamente (9). Otro estudio realizado en la Escuela Militar de Cadetes "General José María Córdova”, que evaluó el estado nutricional de 72 cadetes antes de un Curso Avanzado de Combate, encontró que el $23 \%$ y 4,1 \% de la población presentó un IMC correspondiente a sobrepeso y obesidad, respectivamente (10).

Como se observa, el IMC es la herramienta diagnóstica de mayor uso para definir sobrepeso y obesidad, como exceso de masa grasa. Sin embargo, esta medición no establece diferencia entre masa grasa y masa magra, la cual presenta una amplia variación de acuerdo con el género, la edad, la raza, el nivel de actividad física, entre otros aspectos $(11,12)$.

En este sentido, diferentes estudios han concluido que el uso del IMC por sí solo no es adecuado para evaluar la composición corporal porque reporta resultados de casos falsos negativos o falsos positivos al evaluar la adiposidad corporal $(13,14)$. De acuerdo con la investigación de Carrasco (15), comparado con el Porcentaje de Grasa Corporal (\%GC), el IMC sobreestimó el sobrepeso en el $19 \%$ de los hombres evaluados, al tiempo que subestimó la obesidad en el $23 \%$.

Ante esta evidencia surge la pregunta sobre la pertinencia de la medición del IMC como único estimador del sobrepeso y la obesidad en la población militar, considerando que se trata de una población físicamente activa. Un estudio realizado en el ámbito militar en población chilena reportó que el IMC sobreestimó el sobrepeso en el $23 \%$ comparado con \%GC, pero estimó de manera adecuada la obesidad (16). De igual manera, una investif gación realizada en militares mexicanos encontró que una parte de los individuos que según el IMC habían sido clasificado en sobrepeso eran normales según el Índice de Masa Grasa (17). Por su parte, de acuerdo con Mullie P, 
et al., en población militar americana el IMC entre 25 y 27 tiene una fuerte correlación con aumento de la masa libre de grasa principalmente $(18,19)$.

\section{Otros indicadores antropométricos}

A continuación, se presentan otros indicadores antropométricos que permiten determinar la condición nutricional de una persona.

Circunferencia Abdominal (CA): es uno de los más usados junto al IMC para evaluar la adiposidad corporal. Es un indicador de distribución regional de la grasa y es considerado la medida antropométrica por excelencia para diagnosticar obesidad abdominal, riesgo cardiovascular y síndrome metabólico $(20,21)$ por su asociación con los depósitos de grasa visceral o ectópica. Asimismo, parece ser mejor predictor de adiposidad que el IMC en población joven, de acuerdo con un estudio realizado en población universitaria chilena (22). También se destaca el uso de la circunferencia abdominal, dentro de los estándares de composición corporal de la Armada de los Estados Unidos (la cual estableció su propia fórmula para determinar el $\% \mathrm{GC}$ ), otros investigadores consideran la CA como una de las variables para determinar el sobrepeso y la obesidad $(23,24)$.

Existen diferentes puntos de corte de la CA para clasificar obesidad abdominal. De acuerdo con la OMS, una circunferencia mayor a $94 \mathrm{~cm}$ en hombres y $80 \mathrm{~cm}$ en mujeres se clasifica como preobesidad o riesgo cardiovascular aumentado, mientras que una CA superior a $102 \mathrm{~cm}$ en hombres y $88 \mathrm{~cm}$ en mujeres representa obesidad abdominal y muy alto riesgo de comorbilidad (20). Por su parte, el consenso realizado en 2013 Harmonizing the Metabolic Syndrome, adoptó para población latinoamericana valores de $90 \mathrm{~cm}$ para hombres y $80 \mathrm{~cm}$ para mujeres (25).

Índice Cintura-Altura (ICA): corresponde al valor de CA corregido por la estatura, lo cual le ha dado fuerza predictiva por su asociación con riesgo cardiometabólico (26), con valores superiores a 0,51. Así, y de acuerdo con resultados publicados en el American Journal Clinical Nutrition por Flegan et al. en 2009, el ICA resultó un mejor predictor de adiposidad que el IMC y la CA por sí sola (27). 
Porcentaje de Grasa Corporal (\%GC): el consenso de la Sociedad Española de Endocrinología y Nutrición (Seedo) define como normal un $\%$ GC de entre 12 a $20 \%$ para hombres y 20 a $30 \%$ para mujeres respectivamente en función de los depósitos de grasa corporal. Asimismo, considera límite o sobrepeso 21 a $25 \%$ en hombres y 30 a $33 \%$ en mujeres, en tanto que para la obesidad reporta $>25 \%$ en hombres y $>33 \%$ en mujeres (28). En la tabla 1 se pueden apreciar los rangos de porcentaje de grasa asociados al IMC para el género masculino de acuerdo con la edad (29).

Tabla 1. Porcentaje de grasa para hombres según edad e Índice de Masa Corporal

\begin{tabular}{ccccc}
\hline Edad & Bajo & Normal & $\begin{array}{c}\text { Alto } \\
\text { Sobrepeso } \\
\mathrm{IMC}>\mathbf{2 5} \mathbf{~ k g} / \mathbf{m}^{2}\end{array}$ & $\begin{array}{c}\text { Muy alto } \\
\text { Obesidad Sobrepeso } \\
\mathrm{IMC}>\mathbf{2 5} \mathbf{~ k g} / \mathbf{m}^{2}\end{array}$ \\
\hline $\mathbf{2 0 - 3 9}$ ańos & $<8$ & $<19,9$ & $>20$ & $>25$ \\
$\mathbf{4 0 - 5 9}$ años & $<11$ & $<21,9$ & $>22$ & $>28$ \\
$\mathbf{6 0 - 7 9}$ ańos & $<13$ & $<24,9$ & $>25$ & $>30$ \\
\hline
\end{tabular}

Fuente: Gallagher et al. (29).

Índice de Masa Grasa (IMG): corresponde a la grasa absoluta corregida por la estatura (grasa $\mathrm{kg} / \mathrm{m}^{2}$ ), para el cual no existe un consenso de puntos de corte. De acuerdo con Peine et. al. (30), se consideran normales valores inferiores a $5,60 \mathrm{~kg} / \mathrm{m}^{2}$ en hombres y 7,90 kg / $\mathrm{m}^{2}$ en mujeres, sobrepeso entre $>5,61 \mathrm{~kg} / \mathrm{m}^{2}$ a 7,08 para hombres $\mathrm{y}>7,91$ a 10,30 para mujeres. Para la obesidad da las cifras de $>7,090 \mathrm{~kg} / \mathrm{m}^{2}$ en hombres y $10,31 \mathrm{~kg} /$ $\mathrm{m}^{2}$ en mujeres (30). En otra investigación, un IMG superior a 5,4 kg / $\mathrm{m}^{2}$ en hombres y 7,80 $\mathrm{kg} / \mathrm{m}^{2}$ en mujeres se correlacionaba con sobrepeso y obesidad (31).

Índice de Masa Libre de Grasa (IMLG o FFMI, por su sigla en inglés): corresponde a la Masa Libre de Grasa (MLG) corregida por la estatura $\left(\right.$ FFMI $=$ MLG $\left.(\mathrm{kg}) / \mathrm{m}^{2}\right)$, usado para clasificar individuos con similar composición corporal, pero con diferencia en talla. El IMC clasifica erró- 
neamente a individuos que presentan un exceso de peso a expensas de la masa muscular, y se sugiere que puede ser el caso de un porcentaje de la población militar. Por esto, el FFMI es de especial utilidad en casos de individuos con baja talla y buen desarrollo muscular que el IMC clasifica en sobrepeso y obesidad (32).

\section{Metodología}

Se realizó un estudio observacional de corte transversal. Los datos incluidos dentro de este trabajo provienen de estudiantes activos de las diferentes escuelas de formación militar, como son la Escuela Militar de Suboficiales (Emsub), la Escuela Militar de Cadetes "General José María Córdova” (Esmic) y la Escuela de Soldados Profesionales (Espro). Los datos fueron tomados en las instalaciones de cada una de las escuelas ubicadas en Bogotá y Melgar (Cundinamarca) durante el mes de agosto del año 2018. El total de la muestra correspondió a 137 individuos de género masculino entre los 19 y 31 años de edad. Fueron convocados todos los estudiantes de último nivel de cada una de las escuelas que estuvieran disponibles para el día de la recolección de datos. Los criterios de exclusión para este estudio fueron pertenecer al sexo femenino, no completar todas las variables de medición, no haber cumplido con las recomendaciones de preparación para la prueba de bioimpedancia eléctrica (presentarse en estado de ayuno, no haber realizado actividad física en las doce horas previas, mantener un adecuado estado de hidratación) no firmar el consentimiento informado y tener amputaciones.

Se obtuvo el consentimiento informado por escrito de cada uno de los participantes y el Comité de Ética del Departamento de Investigación, Tecnología y Desarrollo de la Esmic aprobó la intervención siguiendo las normas de la Declaración de Helsinki y la normativa legal vigente en Colombia que regula la investigación en humanos (Resolución 008439 de 1993 del Ministerio de Salud de Colombia). 


\section{Variables de medición}

Se midió estatura, peso y circunferencia de cintura, las cuales fueron registradas por una antropometrísta certificada nivel uno de la International Society for Advancement in Kinanthropometry (ISAK), empleando los siguientes instrumentos de medición: la estatura se midió con un estadiómetro portátil (Seca 206 ${ }^{\circ}$ Hamburgo Alemania) (rango 0-220 cm) de 1 $\mathrm{mm}$ de precisión. Para el peso se utilizó una báscula electrónica de piso (200 $\mathrm{kg}$ ), marca Seca 813, con precisión de 100 gr. La circunferencia de la cintura se midió con una cinta métrica en acero inextensible flexible, marca Lufkin modelo W606PM. La circunferencia de la cintura fue tomada en el punto medio entre el borde inferior de la última costilla y el borde superior de la cresta iliaca.

Para establecer variables como Porcentaje de Grasa, Índice de Masa Grasa, Índice de Masa Muscular y Masa Libre de Grasa se evaluó la compoł sición corporal mediante bioimpedancia eléctrica tetrapolar con el equipo Medical Body Composition Analyzer Seca mBCA 525, siguiendo las recomendaciones del fabricante (no haber consumido comida o bebidas cuatro horas antes de la prueba; no haber realizado ejercicios las doce horas antes del examen; haber orinado treinta minutos antes y no haber ingerido alcohol veinticuatro horas antes). Las valoraciones se realizaron en camilla, posición supina con brazos y piernas ligeramente separados de la línea media.

El Índice Cintura-Altura fue determinado dividiendo la medida de la cintura $(\mathrm{cm})$ por la altura $(\mathrm{cm})$, teniendo como referencia para la discriminación de la obesidad abdominal y riesgo cardiovascular un valor de $\geq 0,51$ (33). Asimismo, para determinar el nivel de adiposidad corporal se tuvieron en cuenta los criterios Seedo 2000 y 2007 descritos anteriormente (28). Para el Índice de Masa Grasa se tuvieron en cuenta los siguientes puntos de corte: normal $<5,60 \mathrm{~kg} / \mathrm{m}^{2}$, sobrepeso $<7,08 \mathrm{~kg} / \mathrm{m}^{2} \mathrm{y}>7,09$, para obesidad (30). Finalmente, para el Índice de Masa Libre de Grasa se consideró normal entre Percentil 25 y Percentil $75(18$ a 19,8 kg / m²) y alta > percentil 90 $>19,9 \mathrm{~kg} / \mathrm{m}^{2}$ (35). La tabla 2 sintetiza las variables y los valores tomados como puntos de corte en la investigación. 
Tabla 2. Variables y puntos de corte

\begin{tabular}{|c|c|c|c|}
\hline Variable & Referencia & Punto de corte & Interpretación \\
\hline \multirow{3}{*}{$\begin{array}{l}\text { Índice de Masa } \\
\text { Corporal (IMC) }\end{array}$} & \multirow{3}{*}{ OMS (4) } & $\begin{array}{l}18,5 \mathrm{~kg} / \mathrm{m}^{2} \text { a } 24,9 \\
\mathrm{~kg} / \mathrm{m}^{2}\end{array}$ & Normal \\
\hline & & $\begin{array}{l}25 \mathrm{~kg} / \mathrm{m}^{2} \text { a } 29,9 \\
\mathrm{~kg} / \mathrm{m}^{2}\end{array}$ & Sobrepeso \\
\hline & & $>30 \mathrm{~kg} / \mathrm{m}^{2}$ & Obesidad \\
\hline $\begin{array}{l}\text { Índice Cintura- } \\
\text { Altura }\end{array}$ & Sánchez et al. (33) & $\geq 0,51$ & $\begin{array}{l}\text { Obesidad abdominal } \\
\text { y riesgo cardiovascular } \\
\text { moderado }\end{array}$ \\
\hline $\begin{array}{l}\text { Circunferencia } \\
\text { Abdominal }\end{array}$ & $\begin{array}{l}\text { Harmonizing } \\
\text { the Metabolic } \\
\text { Syndrome (25) }\end{array}$ & $>90 \mathrm{~cm}$ & $\begin{array}{l}\text { Preobesidad y riesgo } \\
\text { cardiometabólico }\end{array}$ \\
\hline \multirow{3}{*}{$\begin{array}{l}\text { Índice de Masa } \\
\text { Grasa }\end{array}$} & \multirow{3}{*}{ Peine et. al. (30) } & $<5,60 \mathrm{~kg} / \mathrm{m}^{2}$ & Normal \\
\hline & & $\begin{array}{l}>5,61 \mathrm{~kg} / \mathrm{m}^{2} \mathrm{y}<7 \\
, 08 \mathrm{~kg} / \mathrm{m}^{2}\end{array}$ & Sobrepeso \\
\hline & & $>7,09 \mathrm{~kg} / \mathrm{m}^{2}$ & Obesidad \\
\hline \multirow{2}{*}{$\begin{array}{l}\text { Índice de Masa Libre } \\
\text { de Grasa }\end{array}$} & \multirow{2}{*}{$\begin{array}{l}\text { Schutz, Kyle y } \\
\text { Pichard (35) }\end{array}$} & $\begin{array}{l}p 25 \mathrm{y} p 75(18 \mathrm{a} \\
\left.19,8 \mathrm{~kg} / \mathrm{m}^{2}\right)\end{array}$ & Normal \\
\hline & & $\begin{array}{l}>p 90>19,5 \mathrm{~kg} / \\
\mathrm{kg} / \mathrm{m}^{2}\end{array}$ & Alta \\
\hline \multirow{3}{*}{$\begin{array}{l}\text { Porcentaje de Grasa } \\
\text { Corporal }\end{array}$} & \multirow{3}{*}{$\begin{array}{l}\text { Seedo } 2000 \text { y } \\
2007 \text { (28) }\end{array}$} & $12-20 \%$ & Normal \\
\hline & & $21-25 \%$ & Sobrepeso \\
\hline & & $>25 \%$ & Obesidad \\
\hline
\end{tabular}

Fuente: Original de las autoras.

Para el análisis de la información se empleó analítica descriptiva. Con el fin de establecer la media y desviaciones estándar, valores mínimos y máximos, se usó el paquete estadístico SPPSS 25, además se aplicaron pruebas de normalidad por test de Kolmogorov-Smirnov para establecer la normalidad de los datos. Se utilizó test de correlación de Spearman para datos no paramétricos y se estableció la concordancia diagnóstica entre IMC y \%GC, por una parte, y entre IMC y el IMG usando el índice Kappa de Cohen. Finalmente, se calculó la sensibilidad y especificidad del IMC frente al \%GC y el IMG tomadas como el referente gold estándar. 
Los posibles sesgos asociados a esta investigación están relacionados principalmente con el cumplimiento del protocolo para evaluación por bioimpedancia eléctrica, como por ejemplo el hecho de que era muy difícil garantizar que los participantes hubieran cumplido cabalmente con las recomendaciones.

\section{Resultados}

Se analizaron los resultados de 137 individuos del género masculino pertenecientes a las escuelas de formación del Ejército Nacional de Colombia. La distribución por escuela fue la siguiente: Esmic 32,8 \% (n = 45), Emsub $34,3 \%(\mathrm{n}=47)$ y Espro $32,8 \%(\mathrm{n}=45)$. El promedio de edad de la población fue de $22+/-1,8$ años, con un peso promedio de $66,5 \mathrm{~kg}+/-8,04 \mathrm{~kg}$ y con valores mínimos de 46,7 kg y máximos de $90,5 \mathrm{~kg}$. El promedio de IMC fue de $23,3+/-2,22 \mathrm{~kg} / \mathrm{m}^{2}$. El resumen de los demás datos descriptivos para las variables antropométricas analizadas se muestra en la tabla 3 .

Tabla 3. Descriptivos de variables antropométricas en personal en formación de tres escuelas de las Fuerzas Militares de Colombia

\begin{tabular}{|c|c|c|c|c|c|c|c|c|}
\hline & \multirow{2}{*}{$\mathbf{n}$} & \multirow{2}{*}{ Media } & \multirow{2}{*}{$\begin{array}{c}\text { Desviación } \\
\text { estándar }\end{array}$} & \multirow{2}{*}{ Mínimo } & \multirow{2}{*}{ Máximo } & \multicolumn{3}{|c|}{ Percentiles } \\
\hline & & & & & & 25 & 50 & 75 \\
\hline Peso & \multirow{8}{*}{137} & 66,5 & 8,04 & 46,7 & 90,5 & 60,75 & 65,8 & 71,5 \\
\hline Talla & & 1,69 & 0,07 & 1,54 & 1,84 & 1,64 & 1,68 & 1,74 \\
\hline IMC $^{*}$ & & 23,3 & 2,22 & 18,8 & 29,4 & 21,8 & 23,1 & 24,6 \\
\hline$\% \mathrm{GC}^{* *}$ & & 16,4 & 5,63 & 5,9 & 34,7 & 12,4 & 15,7 & 20,5 \\
\hline $\mathrm{IMG}^{* * *}$ & & 3,9 & 1,68 & 1,20 & 9,8 & 2,76 & 3,44 & 4,9 \\
\hline FFMI $^{* * * *}$ & & 19,4 & 1,13 & 16,6 & 22,1 & 18,6 & 19,4 & 20,2 \\
\hline Cintura & & 78,8 & 5,72 & 67,0 & 96,0 & 75,0 & 78,0 & 82 \\
\hline 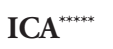 & & 0,47 & 0,04 & 0,40 & 0,60 & 0,44 & 0,46 & 0,49 \\
\hline
\end{tabular}

"Índice de Masa Corporal. "* Porcentaje de Grasa Corporal. ${ }^{* * *}$ Índice de Masa Grasa. ${ }^{* * * *}$ Índice de Masa Libre de Grasa (por su sigla en inglés). ${ }^{* * * *}$ Índice Cintura-Altura.

Fuente: Original de las autoras. 
De acuerdo con el IMC, el 79,5\% $(\mathrm{n}=109)$ se encontraban normales, el 20,4\% ( $n=28)$ con sobrepeso $(n=14)$ y no se reportaron casos de obesidad. Según el \%GC, el 13,1 \% ( $\mathrm{n}=18)$ se clasificaron en sobrepeso y el $8 \%(\mathrm{n}=11)$ en obesidad. Por su parte, el IMG reportó $11,7 \%(\mathrm{n}=16) \mathrm{de}$ sobrepeso y 4,4\% $(n=6)$ de obesidad (figura 1$)$.

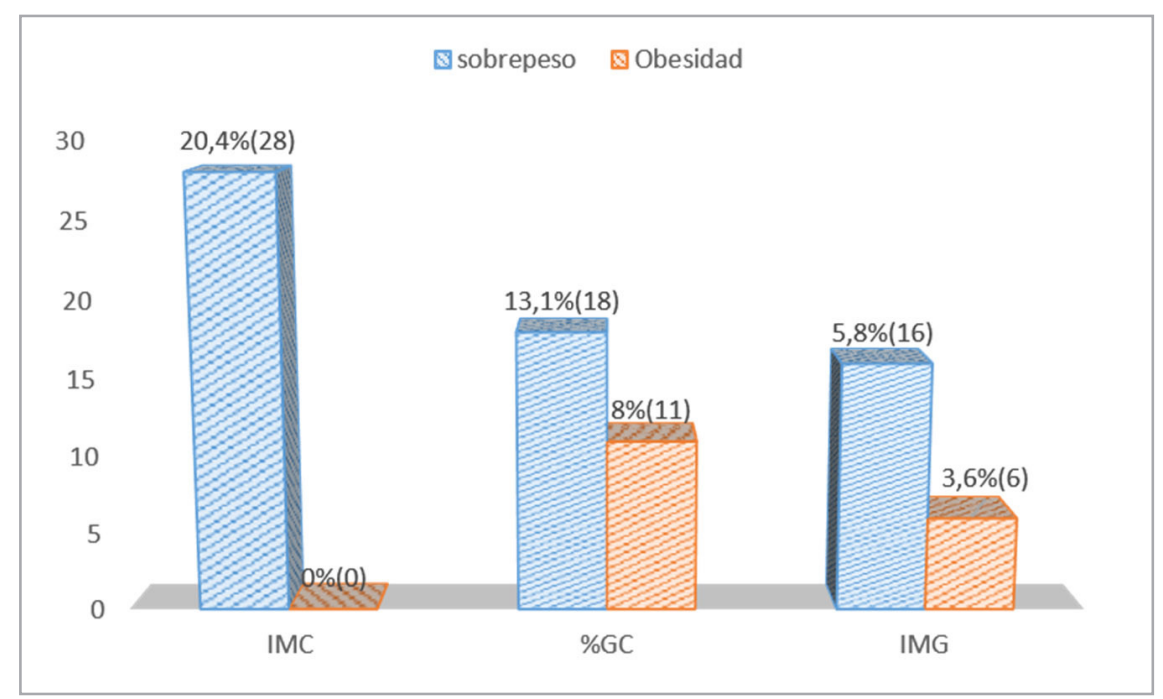

Figura 1. Prevalencia de sobrepeso y obesidad en personal militar en formación de acuerdo con diferentes indicadores: Índice de Masa Corporal $\left(\mathrm{IMC}=\mathrm{kg} / \mathrm{m}^{2}\right)$, Índice de Grasa Corporal (\%GC) e Índice de Masa Grasa $\left(\mathrm{IMG}=\mathrm{kg} / \mathrm{m}^{2}\right)$.

Fuente: Original de las autoras.

De la población con IMC normal, 7 individuos presentaron \%GC y 2 IMG en valores de sobrepeso (falsos negativos). De los 28 individuos clasificados con sobrepeso por IMC, 7 presentaron \%GC normal y 8 presentan IMG normal (falsos positivos). Asimismo, en la clasificación de obesidad el IMC no reportó ningún caso, mientras por \%GC e IMG se presentaron 11 y 6 individuos, respectivamente (falsos negativos para obesidad), como se presenta en las tablas 4 y 5 . 
Tabla 4. Tabla cruzada Índice de Masa Corporal (IMC), según el Porcentaje de Grasa Corporal (\%GC)

\begin{tabular}{llccccc}
\hline & & \multicolumn{4}{c}{ Porcentaje de Grasa Corporal (\%GC) } \\
\cline { 3 - 6 } & & & Normal & Sobrepeso & Obesidad & Total \\
\hline \multirow{3}{*}{ IMC } & \multirow{2}{*}{ Normal } & Recuento & 101 & 7 & 1 & 109 \\
\cline { 3 - 6 } & & \% del total & $73,7 \%$ & $5,1 \%$ & $0,7 \%$ & $79,6 \%$ \\
\cline { 3 - 7 } & \multirow{2}{*}{ Sobrepeso } & Recuento & 7 & 11 & 10 & 28 \\
& & $\%$ del total & $5,1 \%$ & $8,0 \%$ & $7,3 \%$ & $20,4 \%$ \\
\hline \multirow{2}{*}{ Total } & Recuento & 108 & 18 & 11 & 137 \\
& & \% del total & $78,8 \%$ & $13,1 \%$ & $8,0 \%$ & $100,0 \%$ \\
\hline
\end{tabular}

Fuente: Original de las autoras.

Tabla 5. Tabla cruzada Índice de Masa Corporal (IMC), según el Índice de Masa Grasa $\left(\mathrm{IMG}=\mathrm{kg} / \mathrm{m}^{2}\right)$

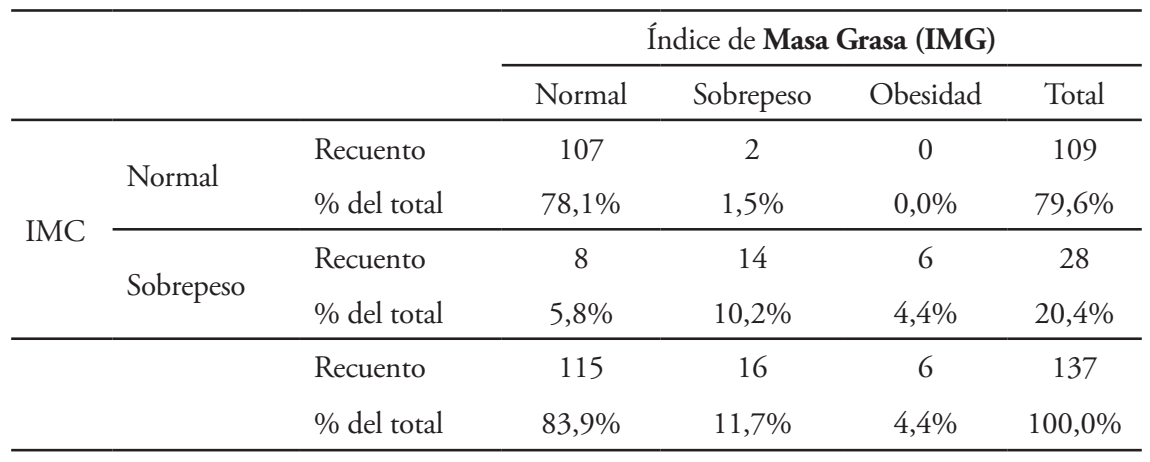

Fuente: Original de las autoras.

Según el FFMI (o IMLG), el 87 \% de la población presenta una reserva de masa magra (MM) normal o alta, así: el 53\% $(\mathrm{n}=73)$ de los individuos se encontraron dentro del percentil 25 a 75 , y $34 \%(n=47)$ sobre el percentil 75. Del total de individuos que presentaron IMC en rangos de sobrepeso, $71 \%$ (20) también presentaron un FFMI por encima del percentil 75.

Respecto a la distribución regional de la grasa, 3,6 \% $(\mathrm{n}=5)$ presentaron una Circunferencia Abdominal (CA) de $>90 \mathrm{~cm}$ o riesgo alto, según criterios de la OMS (4). Por su parte, según el Índice de Cintura-Altura 
(ICA), el 16,7 \% $(\mathrm{n}=23)$ presentaron riesgo cardiometabólico moderado (ICA > 0,51) (figura 2).

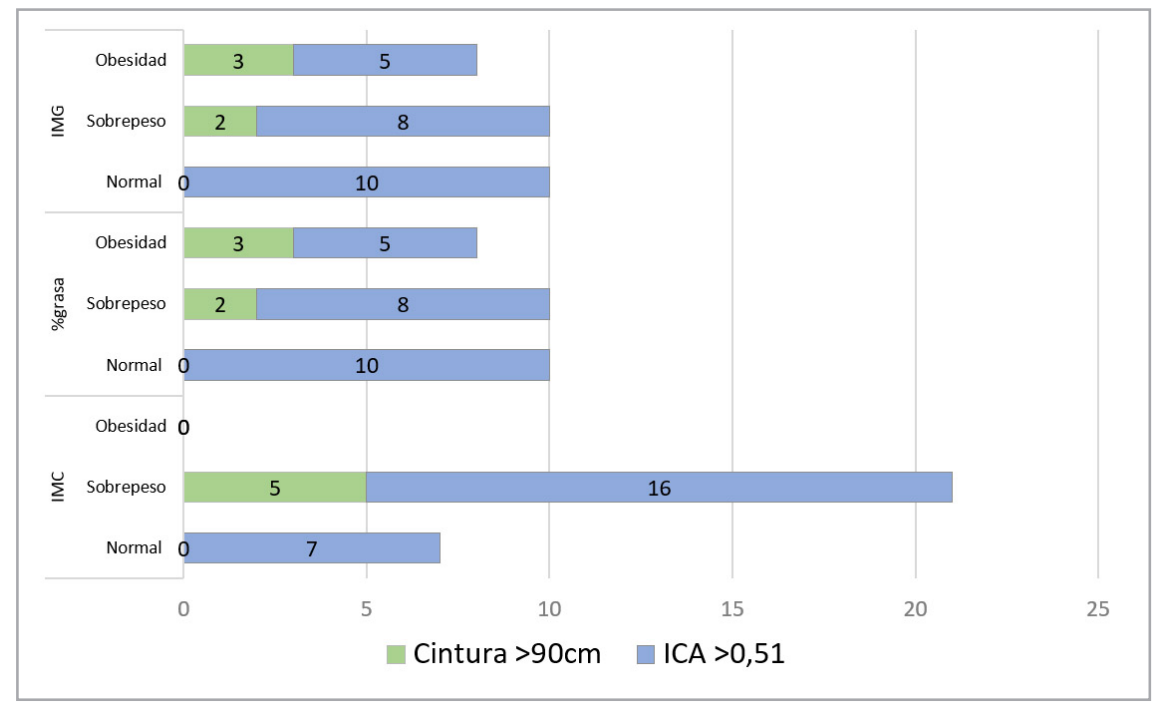

Figura 2. Adiposidad abdominal medida por circunferencia de cintura y corregida por talla para cálculo del Índice de Cintura-Altura, comparado con diferentes indicadores de adiposidad como IMC, \%GC e IMG.

Fuente: Original de las autoras.

En esta población la correlación positiva más significativa fue entre el peso y la masa muscular (MM) y la masa de libre de grasa (MLG) $(r=0,851$, $\mathrm{p}=0,00 \mathrm{y} \mathrm{r}=0,82, \mathrm{p}=0,00$, respectivamente), seguida por el IMC y el IMG $(\mathrm{r}=0,836, \mathrm{p}=0,00)$ y el IMG con el ICA $(\mathrm{r}=0,725, \mathrm{p}=0,000)$ (figuras 3 y 4). También se observó correlación positiva alta entre la talla y la MM y la $\operatorname{MLG}(\mathrm{r}=0,710, \mathrm{p}=0,000$ y r $=0,805, \mathrm{p}=0,000)$, respectivamente. Por su parte, el ICA mostró mejor correlación con el \%GC $(r=0,691, p=0,000)$ y el IMG $(r=0,725, p=0,000)$ que la CA $(r=0,650, p=0,000$ y $r=0,698$, $\mathrm{p}=0,000)($ tabla 6$)$. 


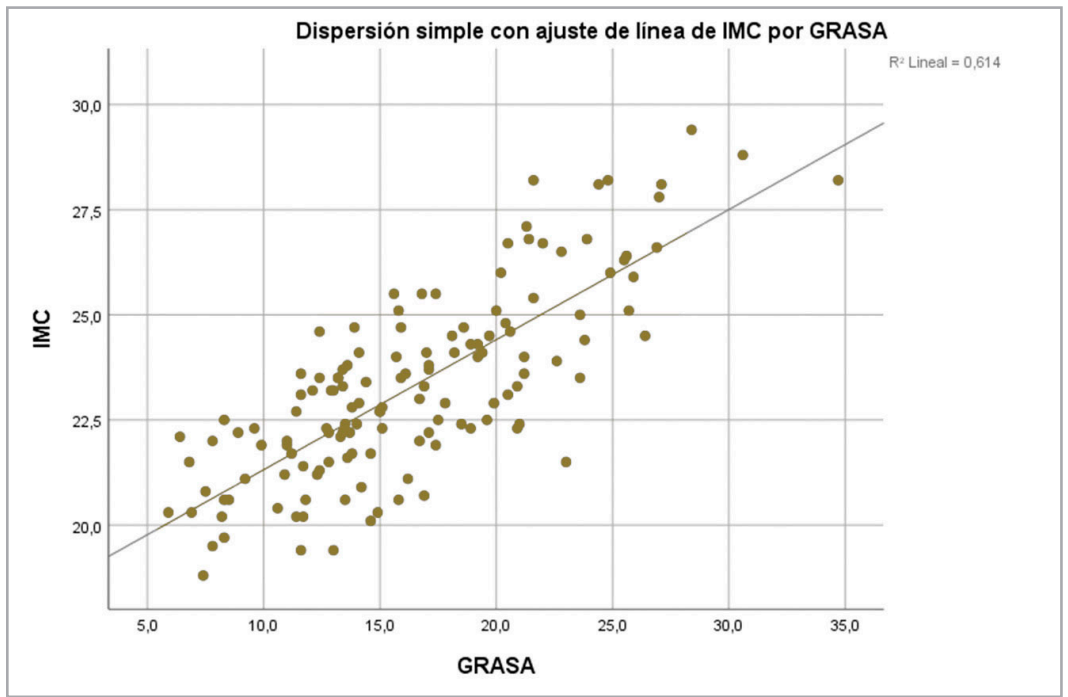

Figura 3. Coeficiente de correlación entre el IMC y el porcentaje de grasa (CC 0,75, $p=$ 0,00, IC $95 \%$ 15,5-17,3 kg / $\mathrm{m}^{2}$ ).

Fuente: Original de las autoras.

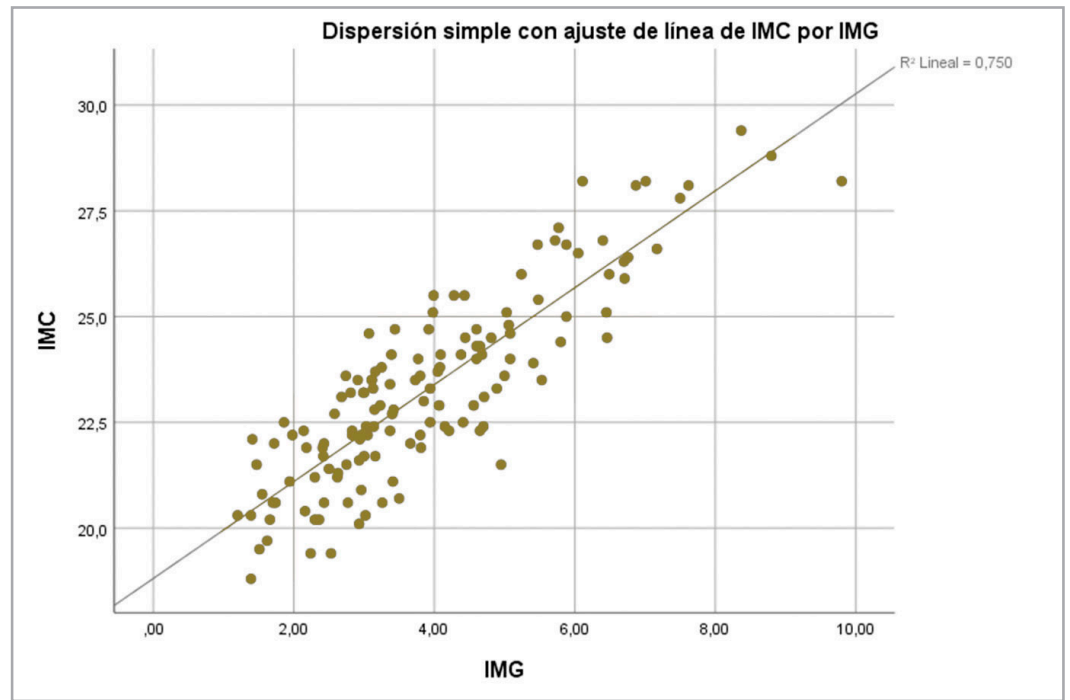

Figura 4. Coeficiente de correlación entre el IMC y el IMG (CC 0,84, $p=0,00$, IC $95 \%$ $\left.3,62-4,18 \mathrm{~kg} / \mathrm{m}^{2}\right)$.

Fuente: Original de las autoras. 


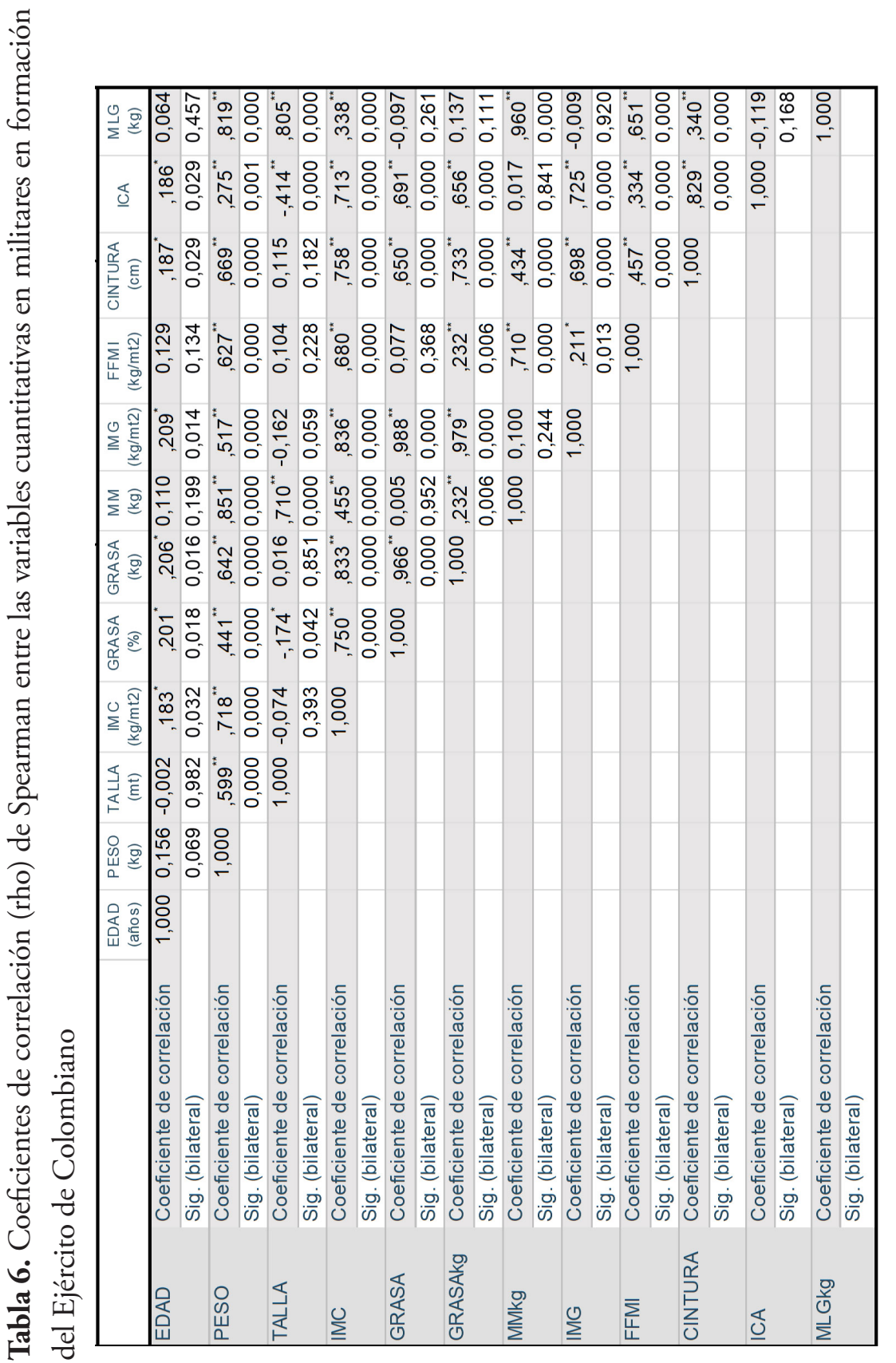

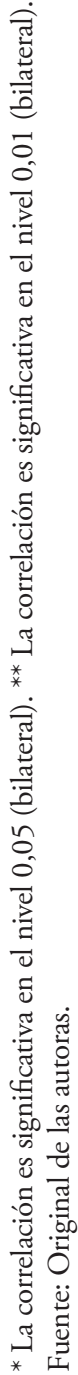


Al evaluar la sensibilidad y especificidad del IMC como prueba diagnóstica de sobrepeso y obesidad, comparado con el \%GC y el IMG como pruebas diagnósticas gold estándar se observó sensibilidad de 0,94 y especificidad de 0,61 para el \%GC en el diagnóstico de sobrepeso. Para obesidad, la sensibilidad y la especificidad fue de 0,94 y 0,91, respectivamente. Además, el IMG (masa grasa corregida por estatura como otra prueba gold estándar para evaluación de adiposidad) mostró una sensibilidad de 0,90 y especificidad de 0,87 para sobrepeso, mientras que presentó sensibilidad y especificidad de 0,91 y 1,00 para obesidad, respectivamente.

El coeficiente Kappa de Cohen para calcular la concordancia diagnósł tica entre el IMC y el \%GC, y el IMC y IMG como predictores de sobrepeso y obesidad fue de k=0,41 para IMC vs $\% \mathrm{GC}$, y de $\mathrm{k}=0,598$ para IMC vs IMG. En las tablas 4 y 5 (supra) se observó la presencia de falsos positivos y negativos para sobrepeso y falsos negativos para diagnóstico de obesidad al compararlos con el IMC.

\section{Discusión}

En la directiva permanente de agosto de 2018 del Ministerio de Defensa Nacional, "Parámetros de evaluación de prueba física para el personal de oficiales y suboficiales de las fuerzas militares" (3), específicamente en el Anexo F, se relacionan los intervalos normales de peso para la estatura de acuerdo con el IMC y los puntos de corte propuestos por la OMS para diagnóstico de sobrepeso y obesidad (4). Sin embargo, a pesar de que el IMC es una herramienta de fácil uso, incurre en errores de predicción del sobrepeso y la obesidad en población militar $(16,17,35)$. En esta investigación, si bien el IMC mostró una correlación significativa tanto con \%GC como con IMG, también se observó que en la población estudiada el IMC frente al \%GC y el ICA subestimó la prevalencia de sobrepeso en 5,1 \% y 1,5 \%, respectivamente, y de obesidad en el $8 \%$ y $4,4 \%$, respectivamente. A su vez, sobreestimó el sobrepeso en 5,1 \% y 5,8 \%, comparado con el \%GC y el ICA, respectivamente. Cabe destacar que los individuos clasificados con 
sobrepeso según IMC y normales según \%GC e ICA se encontraban en un rango de IMC entre 25 y 27, situación observada en otras investigaciones en población militar, en las cuales se sugiere que para esta población los individuos con IMC entre 25 y 27 se debe considerar la composición corporal para discriminar si el sobrepeso es a expensas de la grasa o de la masa magra (19).

Este comportamiento del IMC en la predicción del sobrepeso y la obesidad, comparado con otros indicadores de adiposidad, se confirma con los resultados reportados por Durán-Agüero (16) y Vázquez-Guzmán (17) en población militar chilena y mexicana, respectivamente. Dada esta situación, para que el IMC pueda ser usado como indicador en población militar se deben ajustar los puntos de corte, además se puede establecer el IMG como el mejor indicador de adiposidad para población militar, considerando que presentó la mayor sensibilidad y especificidad, así como el valor Kappa más alto. También se debe señalar que de acuerdo con la investigación de Peine (30) sobre la generación de rangos de normalidad para la composición corporal evaluada mediante bioimpedancia eléctrica comparada con el método de fraccionamiento en cuatro componentes y DEXA, el \%GC sobreestimaba el sobrepeso y la obesidad en comparación con el IMG, situación que es similar a la observada en nuestra investigación. A su vez, se resalta la importancia del uso del ICA como indicador de adiposidad regional por su fuerte correlación con el IMG.

Asimismo, y considerando la masa libre de grasa —el otro componente importante de la composición corporal— uno de los hallazgos más importantes fue la correlación positiva alta entre el peso y la masa muscular y la masa libre de grasa, superior a la correlación entre peso y grasa absoluta. Este resultado sugiere un importante desarrollo muscular en esta población que contribuye a la presencia de IMC más elevados en presencia de niveles de grasa normales, como también identificó Bustamante, quien menciona un desarrollo muscular mayor a la media de la población en cadetes argentinos (34). Situación que no es ajena a nuestro estudio, en el cual se identificaron niveles FFMI superiores al percentil 75 de acuerdo con la tabla publicada por Schutz, Keyle y Pichard (35). 
Por su parte, el índice Kappa de Cohen mostró una concordancia diagnóstica moderada del IMC y el \%GC, así como entre el IMC y el IMG, similar a la reportada por Durán-Agüero (16) en población militar chilena. Esto evidencia que el IMC por sí solo no es una herramienta diagnóstica adecuada para la clasificación nutricional en población militar y que, por lo tanto, es importante incluir otros indicadores de adiposidad para evaluar a los militares en formación en el Ejército Nacional de Colombia.

Finalmente, cabe recordar que dentro de las limitaciones observadas en esta investigación se destaca la dificultad para controlar el cumplimiento cabal de los requisitos para la realización de la bioimpedancia, principalmente el estado de hidratación de la población.

\section{Conclusiones}

Por una parte, el IMC sobreestima el sobrepeso (casos falsos positivos) y subestima la obesidad en población militar (casos falsos negativos). El Índice de Masa Corporal no es un indicador objetivo del estado nutricional de la población militar, por lo cual se recomienda que se use solamente como instrumento de tamizaje y que esté acompańado por otros indicadores como el IMG y el ICA.

Asimismo, es necesario que en futuros estudios con muestras más numerosas se establezcan puntos de corte para IMC acordes con la composición corporal de la población estudiada, considerando que no en todas las escuelas cuentan con equipos para la evaluación de la composición corporal ni tampoco con personal capacitado en técnicas antropométricas.

En síntesis, el Índice de Masa Grasa es mejor indicador de adiposidad en población militar, incluso mejor que el Porcentaje de Grasa Corporal, pues al tener en cuenta la talla, tiene menor posibilidad de sobreestimación o subestimación. Asimismo, se recomienda usar el Índice de Cintura-Altura para la predicción de obesidad abdominal. Sin embargo, estos resultados no se pueden extrapolar a población militar en ejercicio. 


\section{Agradecimientos}

Esta investigación ha sido realizada gracias al financiamiento de la Escuela Militar de Cadetes “General José María Córdova”. No existe ningún conflicto de interés de los investigadores.

\section{Referencias}

1. Castañeda Tovar SM, Caiaffa Bermúdez NS. Relación entre la composición corporal y el rendimiento físico en la Escuela Militar de Cadetes José María Córdova. Revista Cientifica General José María Córdova [Internet]. 2015 [citado 2019 mayo 6]; 13: 257-70. Disponible en: https://doi.org/10.21830/19006586.26

2. Peterson DD. History of the U.S. Navy Body Composition Program. Mil Med 2015; 180(1): 91-6.

3. Ministerio de Defensa Nacional. Parámetros de evaluación de prueba física para el personal de oficiales y suboficiales de las fuerzas militares. 2018.

4. Organización Mundial de la Salud (OMS). Obesidad y sobrepeso [Internet]. 2018 [citado 2019 mayo 6]. Disponible en: https://www.who.int/es/news-room/fact-sheets/ detail/obesity-and-overweight

5. Ministerio de Protección Social, Instituto Colombiano de Bienestar familiar. Encuesta Nacional de Alimentacion y Nutrición (ENSIN) [Internet]. 2015. Disponible en: https:/www.icbf.gov.co/bienestar/nutricion/encuesta-nacional-situacion-nutricional

6. Villatoro Villar M, Mendiola-Fernández R, Alcaraz-Castillo X, Mondragón-Ramírez GK. Correspondencia: Correlación del índice de masa corporal y el porcentaje de grasa corporal en la evaluación del sobrepeso y la obesidad. Rev Sanid Milit Mex [Internet]. 2015 [citada 2019 mayo 6]; 69: 568-78. Disponible en: https://www.medigraphic. $\mathrm{com} / \mathrm{pdfs} / \mathrm{sanmil} / \mathrm{sm}-2015 / \mathrm{sm} 156 \mathrm{i} . \mathrm{pdf}$

7. Ma, V-G, Carrera-Rodríguez, G, Durán García, AB, Gómez-Ortiz, O. Correlación del índice de masa corporal con el índice de masa grasa para diagnosticar sobrepeso y obesidad en población militar. Rev. Sanid. Milit. Mex. [Internet]. 2016 [citado 2019 mayo 6]; 70: 505-15. Disponible en: www.sanidadmilitar.org.mxartículooriginal

8. Teixeira CS, Érico E, Pereira F. Physical fitness, age and nutritional status of milii tary personnel. Arq Bras Cardiol [Internet]. 2010 [citado 2019 mayo 6]; 94: 412-7. Disponible en: http://www.scielo.br/pdf/abc/v94n4/en_aop00110.pdf

9. Fajardo BE, Varela MJ, Castro JJ, Daza CC, Garzon ML, Méndez GM. Caracterización del estado nutricional y la actividad física en una población de pilotos de ala fija rotativa en la ciudad de Bogotá, Colombia. Rev. Med. [Internet]. 2015 [citado 2019 mayo 6]; 23(1): 12-8. Disponible en: http://www.scielo.org.co/pdf/med/v23n1/v23n1a02.pdf 
10. Cortez Fernández S, Camargo IY, Botero RD. Changes in body mass index and composition in active personnel in the Colombian Army: a case study. Rev. Cient. Gen. José María Córdova [Internet]. 2018 [citado 2019 mayo 6]; 16: 93-106. Disponible en: http://dx.doi.org/10.21830/19006586.297

11. National Heart Blood Institute. Managing overweight and obesity in adults systematic evidence review from the Obesity Expert Panel [Internet]. 2013 [citado 2019 mayo 6]. Disponible en: https://www.nhlbi.nih.gov/sites/default/files/media/docs/obesity-evidence-review.pdf

12. Centros para el Control y la Prevención de Enfermedades (CDC). El índice de masa corporal para adultos [Internet]. 2018 [citado 2019 mayo 6]. Disponible en: https:// www.cdc.gov/healthyweight/spanish/assessing/bmi/adult_bmi/index.html

13. Martín J, González G, Rosales G. Relación entre el índice de masa corporal, el porcentaje de grasa y la circunferencia de cintura en universitarios. Investig y Cienc de la Universidad Auton Aguascalientes [Internet]. 2015 [citado 2019 mayo 6]; 65: 26-32. Disponible en: http://www.redalyc.org/articulo.oa?id=67443217004

14. Alberto L, Guzmán C, Andrés Y, Torres M, Alejandro J. Porcentaje de grasa corporal y prevalencia de sobrepeso-obesidad en estudiantes universitarios de rendimiento deportivo de Bogotá, Colombia. Nutr Clín Diet Hosp [Internet]. 2016 [citado 2019 mayo 6]; 36: 68-75. Disponible: http://revista.nutricion.org/PDF/cardozo.pdf

15. Carrasco F, Reyes S. E, Rimler S. O, Ríos C. F. Exactitud del índice de masa corporal en la predicción de la adiposidad medida por impedanciometría bioeléctrica. Arch Latinoam Nutr [Internet]. 2004 [citado 2019 mayo 6]; 54: 81-6. Disponible en: http:// repositorio.uchile.cl/handle/2250/127954

16. Durán-Agüero S, Maraboli Ulloa D, Fernández Frías F, Cubillos Schmied G. Composición corporal en soldados chilenos del Regimiento Buin. Rev. Española Nutr. Humana y Dietética [Internet]. 2017 Apr 4 [citado 2019 mayo 6]; 21(1): 11. Disponible en: http://renhyd.org/index.php/renhyd/article/view/268

17. Vázquez-Guzmán M, Carrera-Rodríguez G, Durán-García A, Gómez-Ortiz O. Correlación del índice de masa corporal con el índice de masa grasa para diagnosticar sobrepeso y obesidad en población militar. Rev. Sanid. Mili. Mex. [Internet]. 2016 [citado 2019 mayo 6]; 70: 505-15. Disponible en: www.sanidadmilitar.org.mxartículooriginal

18. Grier T, Canham-Chervak M, Sharp M, Jones BH. Does body mass index misclassify physically active young men? PMEDR [Internet]. 2015 [citado 2019 mayo 6]; 2: 483-7. Disponible en: http://creativecommons.org/licenses/by/4.0/

19. Mullie P, Vansant G, Hulens M, Clarys P, Degrave E. Evaluation of body fat estimated from body mass index and impedance in Belgian Male Military Candidates: comparing two methods for estimating body composition. Mil. Med. [Internet]. 2008 Mar 1 [citado 2019 mayo 16]; 173(3): 266-70. Disponible en: https://academic.oup.com/ milmed/article/173/3/266-270/4557706 
20. Rosales Ricardo Y. Antropometría en el diagnóstico de pacientes obesos; una revisión. Nutr. Hosp. [Internet]. 2012 [citado 2019 mayo 6]; 27(6): 1803-9. Disponible en: http://scielo.isciii.es/pdf/nh/v27n6/05revision04.pdf

21. Heinrich KM, Jitnarin N, Suminski RR, Berkel L, Hunter CM, Alvarez L, et al. Obesity classification in military personnel: a comparison of body fat, waist circumference, and body mass index measurements. Mil Med Int J [Internet]. 2008 [citado 2019 mayo 6]; 173: 67-73. Disponible en: https://core.ac.uk/download/pdf/10652927.pdf

22. Vilchez C, Cancino CA, Contreras AM, García MM, Roías O, Gómez R, et al. Evaluación de la adiposidad corporal según índice de masa corporal en jóvenes universitarios. Cienc y Enfermería [Internet]. 2017 May [citado 2019 mayo 6]; 23(2): 13-20. Disponible en: http://www.scielo.cl/scielo.php?script=sci_arttext\&pi$\mathrm{d}=$ S0717-95532017000200013\&lng=en\&nrm=iso\&tlng=en

23. Navy U. Guide 4. The Body Composition Assessment (BCA) [Internet]. 2016. Disponible en: https://www.public.navy.mil/bupers-npc/support/21st_Century_ Sailor/physical/Documents/Guide 4- Body Composition Assessment (BCA) 2016.pdf

24. Peterson DD. History of the U.S. Navy Body Composition Program. Mil. Med. [Internet]. 2015 Jan 1 [citado 2019 mayo 6]; 180(1): 91-6. Disponible en: https:// academic.oup.com/milmed/article/180/1/91-96/4159972

25. Alberti KGMM, Eckel RH, Grundy SM, Zimmet PZ, Cleeman JI, Donato KA, et al. Harmonizing the Metabolic Syndrome A Joint Interim Statement of the International Diabetes Federation Task Force on Epidemiology and Prevention; National Heart, Lung, and Blood Institute; American Heart Association; World Heart Federation; International Atherosclerosis Society; and International Association for the Study of Obesity. Circulation [Internet]. 2009 [citado 2019 mayo 6]; 120: 1640-5. Disponible en: http://circ.ahajournals.org

26. Koch E, Romero T, Manríquez L, Taylor A, Román C, Paredes M, et al. Razón cintura-estatura: Un mejor predictor antropométrico de riesgo cardiovascular y mortalidad en adultos chilenos. Nomograma diagnóstico utilizado en el Proyecto San Francisco [Internet]. 2008 [citado 2019 mayo 6]. Disponible en: http://repositorio.uchile.cl/ handle/2250/128451

27. Flegal KM, Shepherd JA, Looker AC, Graubard BI, Borrud LG, Ogden CL, et al. Comparisons of percentage body fat, body mass index, waist circumference, and waiststature ratio in adults. Am J Clin Nutr [Internet]. 2009 Feb [citado 2019 mayo 6]; 89(2): 500-8. Disponible en: http://www.ncbi.nlm.nih.gov/pubmed/19116329

28. Barbany M, Remesar X, Carrillo M, Aranceta J, García-Luna P, Alemany M, et al. Consenso SEEDO'2000 para la evaluación del sobrepeso y la obesidad y el establecimiento de criterios de intervención terapéutica. Med Clin (Barc) [Internet]. 2000 [citado 2019 mayo 6]; 115: 587-597. Disponible en: file:///C:/Users/juan\%20XXIII/ Downloads/S0025775300716320.pdf

29. Gallagher D, Heymsfield SB, Heo M, Jebb SA, Murgatroyd PR, Sakamoto Y. Healthy percentage body fat ranges: an approach for developing guidelines based on body mass 
index. Am J Clin Nutr [Internet]. 2000 Sep 1 [citado 2019 diciembre 11]; 72(3): 694-701. Disponible en: https://academic.oup.com/ajcn/article/72/3/694/4729363

30. Peine S, Knabel S, Carrerol I, Brundert M, Wilhelm J, Ewent A, Denzer U, Jensen B, Lilburn L. Generation of normal ranges for measures of body composition in adults based on bioelectrical impedance analysis using the seca mBCA. Int J Body Compos Res [Internet]. 2013 [citado 2019 mayo 6]; 11:67-76. Disponible en: https://science. seca.com/wp-content/uploads/2017/08/Generation-of-normal-ranges-for-measuresof-body-composition-in-adults-based-on-bioelectrical-impedance-analysis-using-theseca-mBCA.pdf

31. Peltz G, Aguirre MT, Sanderson M, Fadden MK. The role of fat mass index in determining obesity. Am J Hum Biol [Internet]. 2010 [citado 2019 mayo 6]; 22(5): 639-47. Disponible en: http://www.ncbi.nlm.nih.gov/pubmed/20737611

32. Hull H, Thornton J, Wang J, Pierson R Jr, Kaleem Z, et al. Fat-free mass index: changes and race/ethnic differences in adulthood. Int J Obes (Lond) [Internet]. 2011 Jan [citado 2019 mayo 6]; 35(1): 121. Disponible en: http://www.ncbi.nlm.nih.gov/ pubmed/20531353

33. Sánchez A, Muhn M, Lovera M, Ceballos B, Bonneau G, Pedrozo W, et al. Índices antropométricos predicen riesgo cardiometabólico. Estudio de cohorte prospectivo en una población de empleados de hospitales públicos. Rev Argent Endocrinol Metab [Internet]. 2014 [citado 2019 mayo 6]; 51(4): 185-91. Disponible en: http://www. scielo.org.ar/scielo.php?script=sci_arttext\&pid=S1851-30342014000400003\&lng=es

34. Bustamante D, Sbarra J, Yuma F, Laborda R, Mendoza J, Pereira JC, et al. Antropometría y composición corporal. Búsqueda del mejor indicador de sorepeso en el cadete naval. Arch Mil [Internet]. 2011 [cited 2019 May 8];8:55-69. Available from: http://www. ara.mil.ar/archivos/docs/06.bustamante.pdf

35. Schutz Y, Kyle U, Pichard C. Fat-free mass index and fat mass index percentiles in Caucasians aged 18-98. Int J Obes [Internet]. 2002 [citado 2019 mayo 6]; 26: 953-60. Disponible en: www.nature.com/ijo 